\title{
Scientific uncertainty and public policy: Moving on without all the answers
}

Henry N. Pollack, Department of Geological Sciences, University of Michigan, Ann Arbor, Michigan 48109-1005, USA; hpollack@umich.edu

\section{INTRODUCTION}

Science is the foundation of modern society: telecommunications, the Internet, transportation, medicine, public health, agriculture, and much more. As representatives of this cornerstone of modern society, scientists are called upon to inform and advise the elected and appointed officials who formulate public policy. Recent examples of difficult scientific topics and contentious public debate include embryonic stem cells, genetically modified agricultural products, animal and human cloning, radioactive waste disposal, missile defense systems, and anthropogenic climate change. Our National Academies of science and engineering and the Institute of Medicine are responding ever more frequently to requests from Congress to provide up-to-date summaries of the state of the science on a wide array of topics. Repeatedly, the underlying science indicates that all these topics are burdened with uncertainties.

Frequently, "scientific uncertainty" is offered as an excuse to avoid making important policy decisions. We must recognize, however, that delaying decisions because of uncertainty is an implicit endorsement of the status quo and often a thinly veiled excuse for maintaining it. It is a bulwark of the take-no-action policy popularly known as "business as usual."

Discussion of scientific uncertainty in the media and elsewhere should be welcomed by scientists, who are trained to couch their results in terms of the attendant uncertainty. Scientists are encouraged to display data with "error bars" and to frame conclusions in terms of probabilities. While developing public awareness about the nature of scientific uncertainty should in principle be a good thing, in reality it has led to confusion because uncertainty has been both misunderstood and distorted.

\section{UNCERTAINTY AND CLIMATE CHANGE}

Probably no other scientific topic in recent decades has in the eyes of the public been more shrouded in uncertainty than that of global climate change. There are many sources of this puzzlement: some real, some manufactured.

One barrier to understanding is that humans find it difficult to appreciate that we are major players in the natural arena.
Face to face with earthquakes, volcanic eruptions, tsunamis, hurricanes, and tornadoes, the typical human reaction is one of awe and a feeling of personal insignificance. Although an individual may feel helpless when compared to these titanic forces of nature, collectively Earth's human population is staggeringly powerful. Humans are currently the most important geological agents at work on the planet, but many people have a hard time believing it.

Another barrier is the fact that the general public is not well trained in science-too often, the power of many independent lines of evidence goes unappreciated. Confidence in an answer should grow if the same conclusion is reached through independent avenues of research, even if the individual results have greater uncertainties or are on occasion simply wrong. There is a tendency to focus on the weakness of the parts rather than the strength of the whole ${ }^{1}$, supposing that if a single piece of evidence can be discredited, the entire construct will fall like a house of cards. In fact, discrediting a single line of evidence is more like snipping a strand in a net hammock-the hammock continues to be supported by the many strands that remain intact. The scientific evidence for climate change in the natural world is compelling in its totality although individual pieces of the story may indeed be open to some question.

With little skill in understanding science, it should come as no surprise that many Americans are fertile ground in which opinion-shapers may plant seeds of uncertainty, confusion, and doubt. I call these opinion-shapers the "manufacturers and marketers of uncertainty." Their ideological and/or economic interests lie in maintaining the status quo, and they work actively to sow confusion. They tarnish scientific results they don't like with phrases like "unsound science," "junk science," or "uncertain science."

We have seen these manufacturers and marketers of uncertainty in many settings over the years: the agro-chemical industry's obfuscation of the environmental consequences of widespread pesticide use, the tobacco industry's decades-long denial of smoking-related health problems, the electric utility industry's rejection of the role of high sulfur coal in producing acid rain, the leaded gasoline industry's foot-dragging when faced with the deleterious health consequences of environmental lead, the synthetic chemical industry's reaction to the role of CFCs in ozone depletion, and, of course, the fossil fuel industry's long denial of the role of anthropogenic greenhouse gases in climate change.

\footnotetext{
${ }^{1}$ I am grateful to Robert W. Buddemeier, who emphasized these concepts in his review of my book Uncertain Science... Uncertain World, which appeared in Palaios, v. 19, no. 2, p. 188-189, 2004.
} 


\section{Why Not Wait for More Research to Clarify Uncertainty Before Forging Ahead with Policy Decisions?}

This is a legitimate question, but upon analysis and reflection, waiting cannot easily be defended as a course of action. A frequent outcome of conducting more research on complex systems such as planetary climate or terrestrial ecosystems or the human body is that uncertainty, rather than being diminished, actually grows as research reveals an even greater complexity than previously supposed. Additionally, there is an element of wishful thinking (some would say hubris) in imagining that with just a little more research we will find "the right answer" or a "silver bullet" solution.

One must also recognize that much of the uncertainty about how climate will evolve over the twenty-first century will not yield to more research. This irreducible uncertainty is related to demographic, economic, and political developments. The range of unfolding climate pathways that appears in reports from the Intergovernmental Panel on Climate Change is wide indeed, not only because of uncertainties in the climate modeling but also because of uncertainties in world population projections, the economics of alternative non-carbon-based energy development, the degree of integration of the global economy, and the vagaries of international conflicts that impact the production and distribution of carbon-based fuels. These are uncertainties outside the realm of the natural sciences.

Calling for more research must also be seen as a doubleedged sword. Because causes and consequences of environmental problems are typically nonlinear, a decade of delay can have a century of consequence. Moreover, the benefit/cost ratio of remediation is generally greatest when a problem is first recognized. The climate system may also have "tipping points" that, when reached, would have truly catastrophic consequences. Just a few years ago, conventional scientific wisdom held that ice loss from Greenland and West Antarctica was proceeding on a millennial time scale. Recent observations, however, have shown that ice loss is occurring at a much faster pace, as meltwater lubricates the base of ice sheets, and floating ice shelves, which impede the flow of ice from the interior, are rapidly disintegrating. Greenland and West Antarctica each have an ice volume equal to about a seven-meter rise in sea level—waiting for more definitive research results to guide policy formulation is risky business indeed.

\section{FRAMEWORK FOR POLICY FORMULATION IN THE FACE OF AN UNCERTAIN FUTURE}

Policy making must take place in an environment of perpetual uncertainty and cannot wait for clarification that may never come, at least not in a helpful time frame. Therefore, policies adopted in the face of uncertainty should be thought of as provisional, not final. Accurate prediction of the future is difficult, in part because the future is a moving target. That last year's flu shot recipe is not likely to work well this year or next, or that anti-malarial prophylactics of the 1970s are virtually ineffective today, are simple reminders that the future never stands still. Because the future unfolds in unexpected ways, we must be prepared to take small steps, evaluate them continuously, and make mid-course corrections if necessary to get back on track.
As we come to recognize a problem such as acid rain or ozone depletion or global warming, our scientific understanding grows, albeit always remaining incomplete. Clearly, we don't know everything about these phenomena, but that does not mean that we don't know anything. We must not let incomplete knowledge lead to policy paralysis - we must move on without all the answers.

Three simple principles provide a framework for formulating policy under conditions of deep uncertainty. The first is a straightforward acknowledgment that there is a problem to be addressed, that we have incomplete knowledge about the problem, and that as we shape policy on the basis of incomplete knowledge we are likely to make mistakes. This is somewhat akin to the introductory confessions at Alcoholics Anonymous meetings: "My name is John Doe and I am an alcoholic"; if we don't acknowledge a problem, we will never solve it.

The second principle follows directly: because the chances for going astray are relatively high, we need to have many balls in the air at once-we need to think of a multiplicity of pathways forward in order to find one or several that prove helpful. As geologists, we have all had our exposure to T.C. Chamberlin's ${ }^{2}$ exhortation to embrace "multiple working hypotheses" so as not to fall into the rut of confinement that a single hypothesis frequently becomes. In the context of mitigating climate change, we need many horses pulling us toward a greenhouse-stabilized future; e.g., energy conservation, renewable energy sources, and carbon sequestration. That a single "silver bullet" solution will emerge is nothing but wishful thinking.

Third: monitor the future as it unfolds, and make mid-course corrections as necessary. This principle derives from our acknowledgment that we are likely to make some mistakes along the way and policy adjustments may be necessary. We should anticipate the need for and be prepared to make mid-course corrections. This strategy, known as "adaptive management," is one of action coupled with continual evaluation and adaptation to changing circumstances or misguided decisions. Adaptive management is premised on optimism but grounded in skepticism. In the assessment and reevaluation of policy, we must not have unrealistic expectations. A multifaceted policy that does a pretty good job of moving us forward under a range of possible futures is a safer bet than a fantastic solution appropriate only to a very particular future world.

\section{CONCLUSIONS}

We can ill-afford to allow uncertainty to lead to policy paralysis or to be invoked as a reason to maintain the status quo. Scientists should not let science or policy be hijacked by those who, parading beneath a banner of uncertainty, don't like what science is telling them. More research may lead to some better understanding, but uncertainty will never be eliminated-it simply will take on new forms. Long-term solutions will emerge from policies that encourage many incremental and diverse steps and a continuous evaluation of their efficacies. Uncertainty should be recognized not as a barrier to policy formulation and implementation, but rather as a stimulus for creative solutions as it promotes a competition of ideas. 